\title{
Evaluation of the Effect of Irbesartan Combined with PMA on Arrhythmia and Cardiac Function in Patients with Rheumatic Heart Disease
}

\author{
Jinping $\mathrm{Li}^{1}$, Zhongqi Xie ${ }^{2 *}$ \\ ${ }^{1}$ Department of Cardiology, Zhaotong Hospital Affiliated to Kunming Medical University, Zhaotong 657000, Yunnan Province, \\ China \\ ${ }^{2}$ Division of Rheumatology, The First Affiliated Hospital of Kunming Medical University, Kunming 650032, Yunnan Province, \\ China
}

\begin{abstract}
Objective: To investigate the clinical effect of irbesartan combined with potassium magnesium aspartate (PMA) in the treatment of rheumatic heart disease. Methods: A total of 316 patients with

the reconstruction of ventricular function, improve the clinical symptoms of patients, prevent heart failure, and can effectively promote the recovery of cardiac function in patients.
\end{abstract} rheumatic heart disease who were treated in our hospital during the period of March 2018 to January 2021 were selected as the research objects. According to the different treatment methods, they were divided into group A (treatment with irbesartan only) and group B (Using Irbesartan combined with PMA treatment), analyze and compare the cardiac function, diastolic blood pressure, systolic blood pressure, heart rate and clinical treatment effect of the two groups of patients after treatment. Results: After treatment, the left ventricular end-systolic diameter (LVESD), left atrial diameter (LAD), and left ventricular enddiastolic diameter (LVEDD) of the two groups were significantly lower than before treatment, and left ventricular ejection fraction (LVEF) was significantly higher than after treatment, $(P<0.05)$, and the improvement of the above indicators in group B was significantly better than that of group $\mathrm{A},(P<0.05)$. After treatment, the diastolic blood pressure, systolic blood pressure and heart rate of group $\mathrm{B}$ were significantly lower than those of group $\mathrm{A},(P<0.05)$; the total effective rate of treatment in group $\mathrm{B}(87.4 \%)$ was higher than that of group A $(76.3 \%),(P<0.05)$. Conclusion: Irbesartan combined with PMA in the treatment of rheumatic heart disease has a better clinical effect than irbesartan alone. It can promote

Keywords: Rheumatic heart disease; Irbesartan; PMA; Arrhythmia; Cardiac function

Publication date: March, 2021

Publication online: 31 March, 2021

"Corresponding author: Zhongqi Xie, xiezhongqi77 (a)126.com

Rheumatic heart disease is mainly caused by the disease of the heart valve caused by rheumatic fever, which is extremely common in clinical practice. Patients with rheumatic heart disease often have no obvious symptoms in the early stage, and their continued development will cause disease in the patient's mitral valve, causing pulmonary hypertension, heart failure, atrial fibrillation and systemic embolism. In the later stage, patients often show palpitation, shortness of breath, fatigue, cough, and limbs. Edema, coughing up pink foamy sputum, until death of heart failure, and some patients died of arterial embolism and cerebral infarction ${ }^{[1]}$. At present, surgical treatment of the disease is mainly used clinically, but for patients with unknown surgical indications, surgical contraindications, and other reasons that cannot be operated on, they can only be treated with conservative drugs. 


\section{Information and methods}

\subsection{General information}

In this study, 368 patients with rheumatic heart disease who were treated in our hospital during the period from March 2018 to January 2021 were selected as the research object. 52 cases were lost to follow-up in the middle, and 316 patients were finally included. The 93 cases in the A group were treated with irbesartan alone due to contraindications or economic reasons; 223 cases in the B group were treated with irbesartan combined with PMA.
Patient admission criteria were: diagnosed with rheumatic heart disease and heart failure; voluntary conservative treatment, no contraindications to the treatment drugs used in the study, knowledge of the content of this study, and signed informed consent. Exclusion criteria: patients with liver and kidney dysfunction; patients with chronic lung disease; patients with malignant tumors; patients with mental illness; patients allergic to the drugs in this study. The baseline data of the two groups of patients were not statistically different $(P>0.05)$, and they were comparable. See Table 1 for details.

Table 1. C $(\bar{x} \pm s)$

\begin{tabular}{ccccc}
\hline Groups & $\boldsymbol{n}$ & Gender Male/Female & Age Years & Disease duration Years \\
\hline A Group & 93 & $53 / 40$ & $64.5 \pm 4.9$ & $5.3 \pm 1.4$ \\
B Group & 223 & $125 / 98$ & $64.8 \pm 5.2$ & $5.5 \pm 1.8$ \\
\hline
\end{tabular}

\subsection{Methods}

Both groups were given conventional treatments such as anti-infection, sedation, diuresis, and blood pressure control.

\subsubsection{Group A}

Group A was treated with irbesartan alone due to contraindications or economic reasons. Specifications: $150 \mathrm{mg} /$ tablet. Usage and dosage: orally, $150 \mathrm{mg}$ each time, once a day.

\subsubsection{Group B}

Group B was treated with PMA on the basis of the A group. Specifications: $10 \mathrm{ml} /$ piece. Usage and dosage: $40 \mathrm{~mL}$ of potassium and magnesium aspartate, diluted with $250 \mathrm{~mL}$ of $5 \%$ glucose solution, intravenous drip, 1 time/day.

The treatment time for both groups was 6 month. During the entire treatment process, it is necessary to pay close attention to changes in the patient's condition and increase or decrease the amount of medication according to the patient's specific situation.

\subsection{Evaluation indicators}

\subsubsection{Cardiac function}

Evaluate the cardiac function of the two groups of patients before and after treatment. Under the resting state of the patients before and after treatment, the cardiac color Doppler ultrasound was used to measure LVESD, LAD, LVEDD, and calculate LVEF.

\subsubsection{Clinical indicators}

Observe and compare the clinical indicators of diastolic blood pressure, systolic blood pressure and heart rate after treatment between the two groups.

\subsubsection{Clinical efficacy ${ }^{[2]}$}

According to the New York Heart Association (NYHA) cardiac function classification scheme to evaluate the efficacy of patients: Markedly effective: the symptoms are significantly improved, the heart rate reaches the normal standard, and the cardiac function is improved $\geqslant 2$ levels. Effective: the symptoms are improved, the heart rate reaches the normal standard, and the cardiac function is improved by 1 level. Invalid: the symptoms are not improved, the edema is not reduced, and the cardiac function no improvement, even worse. Total effective $=$ Markedly effective + Effective.

\subsection{Statistical methods}

All the data in this study were entered into SPSS 20.0 software for statistical processing. Measurement data such as cardiac function, blood pressure and heart rate of patients are represented by $(\bar{x} \pm s)$, comparison between groups is by t-test. Count data such as gender, total effective rate of treatment are represented by $\%$, and comparison between groups is by $\chi^{2}$ test. Take $P<0.05$ as the difference is statistically significant. 


\section{Results}

2.1 Comparison of changes in cardiac function between two groups of patients before and after treatment

Before treatment, there was no statistical difference in LVESD, LAD, LVEDD and LVEF between the two groups of patients $(P>0.05)$. After treatment, the two groups of LVESD, LAD, LVEDD indicators were significantly lower than before treatment, LVEF was significantly higher than before treatment $(P<0.05)$. After treatment, the LVESD, LAD, and LVEDD indexes of the $\mathrm{B}$ group were significantly lower than those of the A group, and LVEF was significantly higher than that of the A group $(P<0.05)$. See Table 2 for details.

Table 2. $\mathrm{C}(\bar{x} \pm s)$

\begin{tabular}{|c|c|c|c|c|c|c|c|c|c|}
\hline \multirow{2}{*}{ Groups } & \multirow{2}{*}{$n$} & \multicolumn{2}{|c|}{ LVESD(mm) } & \multicolumn{2}{|c|}{ LAD(mm) } & \multicolumn{2}{|c|}{ LVEDD $(\mathrm{mm})$} & \multicolumn{2}{|c|}{ LVEF(\%) } \\
\hline & & Before & After & Before & After & Before & After & Before & After \\
\hline A Group & 93 & $50.7 \pm 10.5$ & $48.3 \pm 9.4^{*}$ & $50.2 \pm 7.6$ & $47.5 \pm 6.9^{*}$ & $61.2 \pm 10.1$ & $57.9 \pm 9.5^{*}$ & $35.5 \pm 8.7$ & $42.6 \pm 9.1^{*}$ \\
\hline B Group & 223 & $50.3 \pm 10.2$ & $44.6 \pm 8.7^{* \#}$ & $49.8 \pm 8.1$ & $44.6 \pm 7.8^{* \#}$ & $60.8 \pm 10.7$ & $52.3 \pm 10.8^{* \#}$ & $35.9 \pm 8.4$ & $48.1 \pm 8.8^{* \#}$ \\
\hline
\end{tabular}

Note: Compared with the same group before treatment, ${ }^{*} P<0.05$; Compared with the A group after treatment, ${ }^{\#} P<0.05$

2.2 Comparison of clinical indicators between two groups of patients after treatment

After treatment, the diastolic blood pressure, systolic blood pressure and heart rate of group B were lower than those of group A. There is a statistical difference $(P<0.05)$. See Table 3 for details.

Table 3. Comparison of clinical indicators $(\bar{x} \pm s)$

\begin{tabular}{ccccc}
\hline Groups & $\boldsymbol{n}$ & $\begin{array}{c}\text { Systolic Pressure } \\
(\mathbf{m m ~ H g})\end{array}$ & $\begin{array}{c}\text { Diastolic Blood Pressure } \\
(\mathbf{m m} \text { Hg) }\end{array}$ & $\begin{array}{c}\text { Heart rate } \\
(\text { times/min) }\end{array}$ \\
\hline A Group & 93 & $119.3 \pm 9.2$ & $82.5 \pm 6.9$ & $88.7 \pm 8.6$ \\
B Group & 223 & $108.8 \pm 8.6^{*}$ & $69.2 \pm 7.7^{*}$ & $76.7 \pm 9.7^{*}$ \\
\hline
\end{tabular}

Note: Compared with the A group, ${ }^{*} P<0.05$

2.3 Comparison of clinical efficacy between the two groups

After treatment, the total effective rate of treatment in group $\mathrm{B}(87.4 \%)$ was significantly higher than that in group A $(76.3 \%),(P<0.05)$, as shown in Table 4.

Table 4. Comparison of clinical efficacy between the two groups $[n(\%)]$

\begin{tabular}{cccccc}
\hline Groups & $\boldsymbol{n}$ & Markedly effective & Effective & Invalid & Total effective \\
\hline A Group & 93 & $40(43.0)$ & $31(33.3)$ & $22(23.7)$ & $71(76.3)$ \\
B Group & 223 & $128(57.4)$ & $67(30.0)$ & $28(12.6)$ & $195(87.4)$ \\
\hline
\end{tabular}

Note: Compared with the A group, $\chi^{2}=6.071, P=0.014$

\section{Discussion}

Rheumatic heart disease is an autoimmune disease. After the disease continues to progress, it is easy to cause a variety of complications. Heart failure is the most common, which seriously threatens the life of patients. Relevant clinical studies have shown that the disease is closely related to neuroendocrine activation, leading to ventricular remodeling ${ }^{[3]}$. Therefore, the key to clinical treatment is to improve abnormal gene synthesis, improve ventricular remodeling, and reduce cardiac load. Clinical medications, such as aldosterone system blockers, $\beta$-receptor blockers, angiotensin II receptor antagonists, etc., improve the patient's hemodynamics, promote ventricular function reconstruction, and achieve corresponding results.

Irbesartan is an angiotensin II (Ang II ) receptor inhibitor, which can effectively inhibit the conversion of Ang I to Ang II , inhibit the release of aldosterone, reduce vasoconstriction, and lower blood pressure. And Ang II receptors are blocked, which can increase plasma renin activity, thereby protecting the heart. The main function of PMA is to improve the energy metabolism of cells, enhance the activities of mitochondrial succinate dehydrogenase and cytochrome oxidase, increase ATP production, enhance the anti-hypoxia ability of the heart, and have a significant promotion effect on the $\mathrm{Na}^{+}-\mathrm{K}^{+}$-ATPase activity of the cell membrane, and promote the entry of $\mathrm{K}^{+}$and $\mathrm{Mg}^{2+}$ into the cell ${ }^{[4]}$. At the same time, the 
protective mechanism of PMA on arrhythmia may involve its anti-oxidative stress effect, and the antioxidative damage mechanism may be mainly related to $\mathrm{Mg}^{2+}$.

In this study, irbesartan and PMA were used in combination therapy. PMA can quickly dissociate from aspartic acid after entering the human body, and aspartic acid has a strong affinity with cells and can be used as a carrier of $\mathrm{K}^{+}$to promote cell metabolism and maintain myocardial contraction, thereby improving myocardial contractility and reducing oxygen consumption. Under hypoxia, it has a protective effect on the heart. The dissociated PMA is the precursor of oxaloacetate in the body and plays an important role in the tricarboxylic acid cycle. Therefore, PMA not only can supplement potassium and magnesium ions, but also promote cell energy metabolism $^{[5]}$.

The results of this study show that irbesartan combined with PMA has a better clinical effect than only irbesartan in the treatment of rheumatic heart disease, which can promote the reconstruction of ventricular function, improve the clinical symptoms of patients, prevent heart failure, improve myocardial blood supply, and promote the recovery of cardiac function in patients.

\section{References}

[1] Cai M, Zhang P. Comparison of therapeutic efficacy and safety of nifedipine and atenolol in the treatment of moderate mitral stenosis in sinus rhythm rheumatic heart disease $[\mathrm{J}]$. China Pharmacy,2017,28(27):3781-3784.

[2] Sjur O, Wiker Y A KB, Fuglesang H FS, et al. Calcar screws and ade-quate reduction reduced the risk of fixation failure in proximal humeral fractures treated with a locking plate: 190 patients followed for a mean of 3years [J]. J Orthop Surg Res, 2018, 13(1): 197-198.

[3] Guo HP, Zhao CX, Jin ZS, et al. Role of potassium magnesium aspartate in preventing postoperative atrial fibrillation after off-pump coronary artery bypass surgery $[\mathrm{J}]$. Chinese Heart Journal,2014,26(1):67-69.

[4] Cao FF, Zhang HT, Zhou HY, et al. Protective effects of potassium magnesium aspartate during perioperative period of cardiac surgery: a meta-analysis $[\mathrm{J}]$. Chinese Journal of Extracorporeal Circulation, 2018,16(3):155-159.

[5] Yang HQ, Xu HT. Irbesartan combined with potassium magnesium aspartate on the heart rhythm of patients with rheumatic heart disease disorders and improvement of heart function $[\mathrm{J}]$. Journal of China Traditional Chinese Medicine Information, 2010,2(12):57. 